\title{
DUB3 deubiquitinates and stabilizes NRF2 in chemotherapy resistance of colorectal cancer
}

\author{
Qi Zhang ${ }^{1} \cdot$ Ze-Yan Zhang ${ }^{1} \cdot$ Huan $\mathrm{Du}^{1} \cdot$ Shang-Ze $\mathrm{Li}^{2} \cdot$ Rongfu Tu${ }^{1} \cdot$ Yi-fan Jia ${ }^{3} \cdot$ Zhe Zheng $^{4} \cdot$ Xue-Min Song $^{5} \cdot$ \\ Run-Lei $\mathrm{Du}^{1} \cdot$ Xiao-Dong Zhang $\mathbb{1 0}^{1}$
}

Received: 10 August 2018 / Revised: 7 January 2019 / Accepted: 30 January 2019 / Published online: 18 February 2019

(c) ADMC Associazione Differenziamento e Morte Cellulare 2019

\begin{abstract}
The transcription factor nuclear factor (erythroid-derived 2)-like 2 (NRF2) is one of the master regulators that control hundreds of genes containing antioxidant response elements (AREs). The NRF2-ARE pathway plays a complex role in colorectal cancer (CRC). NRF2 activity is known to be regulated by KEAP1-CUL3 E3 ligase-mediated ubiquitination, indicating the importance of deubiquitination regulation. However, the deubiquitinase (DUB) of NRF2 remains unknown. Here, by screening a DUB library, we identified DUB3 as a DUB that remarkably stabilized NRF2. Further experiments demonstrated that DUB3 promoted NRF2 stability and transcriptional activity by decreasing the K48-linked ubiquitination of NRF2. Coimmunoprecipitation studies revealed interactions between NRF2 and DUB3, as well as between KEAP1 and DUB3, indicating that NRF2, DUB3, and KEAP1 formed a large functional complex. Importantly, ectopic expression of DUB3 caused NRF2-dependent chemotherapy resistance in colon cancer cell lines. Thus, to the best of our knowledge, our findings are the first to identify DUB3 as a NRF2 DUB and may provide a new strategy against chemotherapy resistance in CRC and other NRF2-related diseases.
\end{abstract}

These authors contributed equally: Qi Zhang, Ze-Yan Zhang

Edited by D. Guardavaccaro

Supplementary information The online version of this article (https:// doi.org/10.1038/s41418-019-0303-z) contains supplementary material, which is available to authorized users.

Ze-Yan Zhang

zhangzy@whu.edu.cn

$\triangle$ Run-Lei Du

runleidu@whu.edu.cn

$\triangle$ Xiao-Dong Zhang

zhangxd@whu.edu.cn

1 Hubei Key Laboratory of Cell Homeostasis, College of Life Sciences, Wuhan University, Wuhan 430072, P. R. China

2 Medical Science Research Center, Zhongnan Hospital of Wuhan University, Wuhan, Hubei 430071, China

3 Renmin Hospital of Wuhan University, Hubei General Hospital, Wuhan 430072, P. R. China

4 Clinic Center of Human Gene Research, Union Hospital, Tongji Medical College, Huazhong University of Science and Technology, Wuhan 430000, P. R. China

5 Research Centre of Anesthesiology and Critical Care Medicine, Zhongnan Hospital of Wuhan University, Wuhan, Hubei 430071, P. R. China

\section{Introduction}

Colorectal cancer (CRC) is the third most commonly diagnosed cancer among both men and women worldwide, causing more than half a million deaths per year $[1,2]$. Many researchers have attempted to unravel the complex role of NRF2 in CRC. Some researchers have proven that the induced expression of NRF2 confers protective effects on CRC by decreasing genotoxic damage [3], reducing cell proliferation and increasing apoptosis $[4,5]$. In other cases, constitutive activation of NRF2 promotes a series of events that lead to an increased risk of CRC, such as colonic inflammation [6], uncontrolled cell proliferation [7], and angiogenesis [8]. 5-Fluorouracil (5-FU) [9] and camptothecin (CPT) [10] are the most common chemotherapeutic agents used for the treatment of CRC. Unfortunately, multidrug resistance remains one of the main obstacles to efficient chemotherapy of CRC [11]. Several studies have revealed the relation between NRF2 overexpression and increased resistance to 5-FU in CRC $[12,13]$. Resistance to paclitaxel in mammary cancer was also found to be related to NRF2 overexpression [14]. In this study, we focused on the molecular mechanism of resistance to camptothecin and paclitaxel in CRC cells. Paclitaxel is not routinely used to 
treat CRC now, but several clinical trials are ongoing. (https://clinicaltrials.gov/ct2/show/NCT02103062)

NRF2 is a basic region-leucine zipper (bZIP) transcription factor that functions as a master regulator of cellular homeostasis. NRF2 regulates a battery of downstream genes by recognizing enhancer sequences called antioxidant response elements (AREs) in their promoters [15]. These ARE-genes encode a network of cooperating enzymes that participate in various pathophysiological processes, such as heme oxygenase (HO-1) and glutamate cysteine ligase (GCL) in redox balance [16], macrophage receptor with collagenous structure (MARCO) in inflammation [17], carboxylesterase 1G (CES1G) in lipid metabolism [18], and multidrug resistance-associated proteins (MRPs) in chemotherapeutic drug transport [19]. Given the extensive cellular functions of ARE-genes, NRF2 participates in the genesis and progression of many chronic diseases, including inflammation, autoimmune diseases, chronic respiratory diseases, digestive dysfunction, cardiovascular diseases, metabolic diseases, and neurodegenerative diseases [20]. However, debate continues regarding whether the activation or, alternatively, the inhibition of NRF2 is a useful strategy for preventing or treating chronic diseases and cancer [21]. Therefore, it is imperative to investigate the basic molecular mechanisms of the regulatory network of NRF2 so that we can target these regulators to help prevent and treat associated diseases, including cancer.

Under homeostatic or unstressed conditions, the NRF2 protein level and transcriptional activity are relatively low. Three E3 ubiquitin ligase complexes control the ubiquitination and proteasomal degradation of NRF2: KEAP1CUL3-RBX1， $\beta$-TrCP-SKP1-CUL1-RBX1, and HRD1 [22]. The prominent regulatory mechanism, KEAP1 binds to CUL3 via the N-terminal BTB domain and binds to NRF2 through the C-terminal Kelch domain; thus, KEAP1 is a homodimeric substrate adaptor protein that bridges NRF2 to CUL3 [23-25]. The majority of known physiological or pharmacological NRF2 inducers inhibit KEAP1 in two different ways [26]. One is the loss of KEAP1 protein secondary structure, making KEAP1 unable to bind to NRF2. The other is prevention of the interaction between KEAP1 and CUL3/RBX1, precluding NRF2 degradation $[27,28]$. KEAP1 has been reported to undergo Lys-63 polyubiquitination and to be degraded independent of the $26 \mathrm{~S}$ proteasome [29]. A deubiquitinase, USP15, has been found to deubiquitinate KEAP1 and indirectly aggravate NRF2 Lys-48-linked polyubiquitination, thus decreasing NRF2 protein level [14]. Although the E3 ubiquitin ligase has been very well studied [30-32], the DUBs of NRF2 remain largely unknown. Consequently, identifying the DUB(s) of NRF2 will make it easier and more effective to explore the regulatory network of NRF2-ARE signaling.
DUB3, a member of the ubiquitin-specific processing proteases (UBP or USP) family, has been identified as a novel human deubiquitinating enzyme induced in response to cytokine stimulation [33]. DUB3 belongs to the highly conserved human DUB/USP17 family [34], which resembles four murine homologs named DUB-1, DUB-1A, DUB-2, and DUB-2A [35-38]. DUB/USP17 family members are encoded by a human mega satellite tandem repetitive sequence (RS447) in a unique expression pattern in which the complementary strand is transcribed as an antisense transcript [39]. Cys89 in DUB3 functions as the deubiquitinating catalytic active site, and its mutation to Ser (DUB3 ${ }^{\mathrm{C} 89 \mathrm{~S}}$ ) blocks the deubiquitinating catalytic activity [40, 41]. Recently, accumulating evidence has highlighted the important function of DUB3 in multiple cellular processes and diseases. DUB3 controls the DNA damage response by deubiquitinating H2AX [42] and DEC1 [43]. DUB3 inhibits virus-induced type I IFN signaling by deubiquitinating RIG-I and MDA5 [44]. Moreover, DUB3 stabilizes the ITCH, LATS, and AMOT proteins to regulate Hippo signaling [45]. Genomic analysis and tumor biopsies suggest that DUB3 is upregulated in several cancers, including human breast cancer [46, 47], lung cancer [48], epithelial ovarian cancer [49], and osteosarcoma [50]. The high protein level of DUB3 in tumors regulates cell proliferation, metastasis, and epithelialmesenchymal transition, indicating that DUB3 is a potential therapeutic target for treating cancer.

In this study, we identify DUB3 as the first DUB that directly targets NRF2. DUB3 decreases the NRF2 K48ubiquitination level and promotes NRF2 stability by forming a complex with NRF2 and KEAP1. More importantly, we reveal the molecular mechanism via which DUB3 promotes chemotherapy resistance by promoting NRF2 stability in colon cancer. Our study provides novel insights into the protein homeostasis of NRF2 in tumorigenesis.

\section{Materials and methods}

\section{Antibodies and reagents}

Mouse monoclonal antibodies against GAPDH (cat\# CW0266A, Beijing Cowin Biotech), Flag (cat\# M185-3L, MBL), Myc (cat\# M192-3, MBL), HA (cat\# M180-3, MBL), and ubiquitin (cat\# 646302, Biolegend); a rabbit monoclonal antibody against NRF2 (D1Z9C) (cat\# 12721, Cell Signaling Technology); rabbit polyclonal antibodies against USP17L (S-14) (cat\# sc-103318, Santa Cruz Biotechnology), KEAP1 (cat\# A1820, ABclonal), HMOX1 (cat\# A1346, ABclonal); the proteasome inhibitor MG132 (cat\# S2619, Selleckchem); the protein translation inhibitor cycloheximide (CHX, cat\# C7698, Sigma); paclitaxel (cat\# S1150, Selleckchem); camptothecin (CPT, cat\# HY-16560, 
MedChem Express), and luciferase reporters (Madison) were purchased from the indicated manufacturers.

\section{Tissue samples}

CRC specimens and adjacent tissues were obtained from Tongji Medical College of Huazhong University of Science and Technology (Wuhan, China). All the samples had a clear histologic diagnosis of CRC assessed by experienced pathologists. All samples were obtained from patients who had not received chemotherapy or radiotherapy prior to the operation. The protocols for human studies were in accordance with the Institute Research Medical Ethics Committee of Huazhong University of Science and Technology, and each patient signed individual consent forms.

\section{Cell culture}

Human CRC cell lines including HCT116, SW48, SW480, RKO, DLD1, LOVO, HT29, the human normal colon cell line FHC, the human embryonic kidney 293T cell line (HEK293T), and the human osteosarcoma cell line U2OS were purchased from China Center for Type Culture Collection (CCTCC). HEK293T and U2OS cells were grown in Dulbecco's modified Eagle's medium (HyClone, Logan, Utah, USA) supplemented with $10 \%$ fetal bovine serum (Gibco, Carlsbad, CA, USA) and $1 \%$ penicillinstreptomycin (HyClone, Logan, Utah, USA) at $37^{\circ} \mathrm{C}$ in $5 \% \mathrm{CO}_{2}$. FHC and the $\mathrm{CRC}$ cell lines were grown in McCoy's 5A medium (AppliChem, Darmstadt, Germany) supplemented with $10 \%$ fetal bovine serum and $1 \%$ penicillin-streptomycin at $37{ }^{\circ} \mathrm{C}$ in $5 \% \mathrm{CO}_{2}$.

\section{Plasmids and shRNAs}

Plasmids expressing NRF2-Myc and NRF2-HA were generated using PCR and cloned into pCDNA5/FRT/TO-Myc and pCDNA5/FRT/TO-HA, respectively. DUB3-Flag and DUB ${ }^{\mathrm{C} 895}$-Flag were generated using PCR and cloned into pCDNA5/FRT/TO-Flag. KEAP1-HA and luciferase-HA (lucHA) were generated by PCR and cloned into pCDNA5/FRT/ TO-HA. NRF2-Flag and DUB3-Flag were generated by PCR and cloned into pHAGE-CMV-MCS-PGK-puro. pRL-TK and pGL3-NRF2-luc were purchased from Addgene (Cambridge, MA, USA). Ubiquitin48-HA was kindly provided by Bo Zhong (Wuhan University, Wuhan, China). Doublestranded oligonucleotides corresponding to the target sequences were cloned into pLKO.1 (Addgene, \#8453). The shRNA hairpin sequences were as follows: shNRF2-1, 5'AGAGCAAGATTTAGATCATTT-3'; shNRF2-2, 5'-GCTC CTACTGTGATGTGAAAT-3'; shDUB3-1, 5'-CAAGAAG GCCAGTGGTATAAA-3'; shDUB3-2, 5'-GCCTGAGTTC AACGTCAGAAA-3'.
Transient transfections and lentivirus-mediated stable transfections

Transient transfections were performed with polyethylenimine (PEI; Polysciences) according to the manufacturer's instructions. For lentivirus production, HEK293T cells were transfected with pHAGE or pLKO.1 and the packing vectors pMD2.G (Addgene, \#12259) and psPAX2 (Addgene, \#12260). Virus-containing medium was collected $60 \mathrm{~h}$ after transfection and filtered with a $0.45-\mu \mathrm{m}$ filter (Nalenge, Rochester, NY, USA). Viral supernatant mixed with $8 \mu \mathrm{g} / \mathrm{mL}$ polybrene was used to infect the target cells for $6 \mathrm{~h}$ and was then replaced with fresh medium. The stable cell lines were selected with treatment of $2 \mu \mathrm{g} / \mathrm{mL}$ puromycin $48 \mathrm{~h}$ after transfection. We produced the lentiviruses containing pHAGE-control, pHAGE-NRF2, and pHAGE-DUB3 to establish stable cell lines. The controlshRNA (shNC), NRF2-shRNA, and DUB3-shRNAs lentiviruses were purchased to establish knockdown cell lines. The efficiency in different cells was determined by western blot.

\section{CRISPR/Cas9 knockout (KO) cell line}

The NRF2 knockout HCT116 cell line used a CRISPR/ Cas9 system according to the standard protocol provided by Zhang's lab [51]. Briefly, single-guide RNA (sgRNA) was designed using the online CRISPR Design Tool (http://tools. genome-engineering.org) and cloned into the plasmid lentiCRISPRv2 (Addgene, \#52961). The sgRNA sequence of NRF2 was 5'-TAGTTGTAACTGAGCGAAAA-3'. Lentivirus was produced with the packing vectors pMD2.G and psPAX2 in HEK293T cells. NRF2 knockout HCT116 cell lines were selected by puromycin 2 days after lentivirus infection. A total of $1 \times 10^{5}$ selected cells were split into six 96-well plates to obtain as many single clones as possible. Positive single clones were identified by western blot and PCR. Two different NRF2knockout cell lines were used in subsequent experiments.

\section{Reporter assays}

Using a dual-luciferase assay kit (Promega), reporter assays were performed $24 \mathrm{~h}$ after cells were transfected with pGL3NRF2-luc (containing the repetitive ARE sequence), pRLTK and the indicated levels of the expression constructs.

\section{Western blot}

Cells were washed by ice-cold PBS $36-48 \mathrm{~h}$ after transfection, lysed in RIPA buffer (1\% NP-40, $0.5 \%$ sodium deoxycholate, $0.1 \%$ SDS, $10 \mu \mathrm{g} / \mathrm{mL}$ aprotinin, $10 \mu \mathrm{g} / \mathrm{mL}$ leupeptin, and $1 \mathrm{mM}$ phenylmethylsulfonyl fluoride) in an ice bath for $30 \mathrm{~min}$, and then centrifuged at $13,000 \times \mathrm{g}$ for 
$30 \mathrm{~min}$ at $4{ }^{\circ} \mathrm{C}$. The protein concentration was determined using a BCA protein assay kit (Thermo). Equal amounts of protein $(30 \mu \mathrm{g})$ were separated by $10 \%$ SDS-PAGE and transferred to polyvinylidene difluoride membranes (PVDF, Millipore, cat\# IPVH00010, Merck KgaA, Darmstadt, Germany). The membranes were blocked with $5 \%$ nonfat dry milk in TBST for $30 \mathrm{~min}$ at room temperature, probed with specific primary antibodies overnight at $4{ }^{\circ} \mathrm{C}$, and incubated with an HRP-conjugated secondary antibody for $2 \mathrm{~h}$ at room temperature. Finally, the bands were visualized by a SuperSignal chemiluminescence kit (Merck Millipore).

\section{Coimmunoprecipitation}

Cells were lysed in NP-40 lysis buffer $(30 \mathrm{mM}$ Tris- $\mathrm{HCl}$ (pH 7.4), $150 \mathrm{mM} \mathrm{NaCl}, 1 \% \mathrm{NP}-40,10 \mu \mathrm{g} / \mathrm{mL}$ aprotinin, $10 \mu \mathrm{g} / \mathrm{mL}$ leupeptin, and $1 \mathrm{mM}$ phenylmethylsulfonyl fluoride). Cell lysates were incubated with the indicated antibody and protein G-Agarose beads (Roche) at $4{ }^{\circ} \mathrm{C}$ for $2 \mathrm{~h}$ or overnight. Then, the beads were washed three times with $1 \mathrm{~mL}$ wash buffer containing $300 \mathrm{mM} \mathrm{NaCl}$ at $4{ }^{\circ} \mathrm{C}$. The precipitates were analyzed by standard western blot.

\section{RNA isolation and quantitative real-time PCR (qPCR)}

Total RNA was isolated using TRIzol reagent (TaKaRa Biotechnology, Dalian, China), and the cDNA was reverse-transcribed using the First Strand cDNA Synthesis Kit (Roche Diagnostics, Mannheim, Germany). Quantitative PCR was performed on an ABI PRISM 7500 system (Applied Biosystems, Forster City, Calif) by using the FastStar Universal SYBR Green Master protocol (ROCHE, 04913850001). Target mRNA levels were normalized to GAPDH mRNA. The following primers were used for qPCR: NRF2, 5'-TCAGCGACGGAAAGA GTATG-3' (forward) and 5'-GGGCAACCTGGGAGTA GTT-3' (reverse); DUB3, 5'-CCCTGCTAAACCTCTCT TCG-3' (forward) and 5'-AGAGCCCTCTTGCTGTGT TT-3' (reverse); HO-1, 5'-CAGTCAGGCAGAGGGTGA TA-3' (forward) and 5'-GGCAGAATCTTGCACTTTG TT-3' (reverse); MRP2, 5'-TGCTGAAATTGCTGATCT CC-3' (forward) and 5'-GCTTGAAGCACAGTTGGAA A-3' (reverse); and GAPDH, 5'-GAGTCAACGGATTTG GTCGT-3' (forward) and 5'-GACAAGCTTCCCGTTCT CAG-3' (reverse).

\section{Protein half-life analysis}

Cells were treated with $\mathrm{CHX}(50 \mu \mathrm{M})$ at the indicated time points $48 \mathrm{~h}$ after transfection. Cell lysates were analyzed using standard western blot.

\section{Denaturing immunoprecipitation and ubiquitination analysis}

Denaturing immunoprecipitation and ubiquitination analysis were performed as previously described [52].

\section{Cell viability assay}

The cell viability assay was performed as previously described [53]. Cell Counting Kit-8 (CCK-8) assays were performed to assess cellular proliferation. Cells $\left(1 \times 10^{5}\right)$ were seeded in a 96-well plate and treated with or without chemotherapy drugs for $48 \mathrm{~h}$ on the following day. Then, the medium was replaced with $100 \mu \mathrm{L}$ fresh medium containing $10 \%$ CCK-8 reagent. One hour later, the absorbance was measured at $450 \mathrm{~nm}$ using a microplate reader (ELx800; BioTek, Winooski, VT, USA).

\section{Colony formation assay}

The colony formation assay was performed as previously described [53]. Cells $\left(1 \times 10^{3}\right)$ were seeded in a six-well plate and treated with or without paclitaxel for $48 \mathrm{~h}$ on the following day, after which the medium was replaced with fresh medium. Clones were stained with crystal violet and photographed 10 days later.

\section{Statistical analyses}

Statistical analyses were performed using SPSS software, and the data are expressed as the mean \pm SD. Statistical differences among more than two groups were compared using a one-way analysis of variance, followed by Bonferroni's post hoc test (assuming equal variances) or Tamhane's T2 post hoc test. Student's $t$ test was performed to compare the differences between two groups. $P<0.05$ was considered significant.

\section{Results}

\section{Identification of DUB3 as a potential DUB of NRF2}

It has been well documented that NRF2 is a master regulator of the cellular antioxidant response related to both tumor-promoting and tumor-suppressive effects in the hallmarks of cancer [22]. The NRF2 level is tightly regulated by the ubiquitin-proteasome system [54]. Although the E3 ligases of NRF2 have been well studied, the DUB is still unknown. To identify the functional DUB(s) targeting NRF2, we coexpressed Myc-tagged NRF2 with 66 Flagtagged DUBs, respectively, in HEK293T cells and determined whether any of these DUBs effectively blocked 
NRF2 degradation. Luc-HA was cotransfected in these cells to confirm transfection efficiency. The NRF2-Myc protein level was normalized to luc-HA. The screening results showed that DUB3 was the most potent candidate that increased the NRF2 protein level (Supplementary Information, Fig. S1). Western blot (Fig. 1a) and luciferase assay (Fig. 1b) were conducted to verify the effects of the top 10 DUBs on NRF2. The results suggested that DUB3 stood out as the top candidate for a possible NRF2 deubiquittinase.

\section{NRF2 is upregulated in colon cancer samples and correlated with DUB3}

To determine the clinical significances of NRF2 in patients with CRC, we performed data mining and analyzed NRF2 expression from the publicly available Oncomine database [55]. NRF2 gene expression had a significant $P$-value $(P<$ 0.001 ) in three databases and ranked in the top $10 \%$ among all differentially expressed genes. In the three databases, $N R F 2$ was upregulated in CRC tumor tissues compared with normal tissues (Fig. 1c). To further confirm this conclusion, 24 matched pairs of human CRC and adjacent nontumor tissues were selected for western blot. The results showed a strong correlation between NRF2 and DUB3 in human CRC tissues (Fig. 1d). Additionally, we determined the protein levels of NRF2 and DUB3 in the human embryonic kidney cell line HEK293T, the normal human colon cell line FHC and seven colorectal cell lines (HCT116, SW48, SW480, RKO, DLD1, LOVO, and HT29). The results further confirmed that both NRF2 and DUB3 were overexpressed in CRC cell lines and HCT116 and RKO had relative higher expression levels (Fig. 1e). Therefore, the further molecular mechanism experiments were conducted in HCT116 and RKO cells.

\section{DUB3 stabilizes the NRF2 protein}

To further evaluate whether DUB3 regulates the endogenous NRF2 pathway, immunoblot analysis was performed to determine the protein expression of NRF2, its downstream gene HO-1 and its E3 adaptor KEAP1. HCT116 cells were transfected with an empty vector or the pcDNA5-DUB3Flag expression plasmid in the indicated amounts. The results showed that overexpression of DUB3 upregulated the protein levels of NRF2 and its downstream gene, HO-1, in HCT116 cells in a concentration-dependent manner (Fig. 2a). However, KEAP1 was free of the effect (Fig. 2a). To examine the regulation of NRF2 in a more physiological context, we knocked down DUB3 using two different effective shRNAs in HCT116 cells. As expected, use of the DUB3-shRNAs (shD-1 and shD-2) resulted in a significant decrease in the protein expression of NRF2 and HO-1 and no effect on KEAP1 (Fig. 2b). Wild-type DUB3 and a catalytic mutant with Cys89 mutated to Ser (DUB3 ${ }^{\mathrm{C} 89 \mathrm{~S}}$ ) were used to confirm that the effect was dependent on the catalytic activity of DUB3. In HCT116 cells, overexpression of the wild-type DUB3, but not DUB3 ${ }^{\mathrm{C} 89 \mathrm{~S}}$, substantially increased the NRF2 protein level (Fig. 2c), indicating that NRF2 was regulated by DUB3 in a deubiquitinating enzyme activity-dependent manner. To further verify that the observed changes in NRF2 protein level in response to DUB3 overexpression or interference did not result in a change in NRF2 transcription, the NRF2 mRNA level was measured by real-time PCR. As expected, the $N R F 2$ mRNA level remained unchanged in the presence or absence of DUB3 (Fig. 2d). We then examined endogenous NRF2 protein level in the presence of CHX, an inhibitor of protein translation. Remarkably, overexpression of DUB3 but not DUB3 ${ }^{\mathrm{C} 89 \mathrm{~S}}$ led to a noticeable increase in the stability of NRF2 (Fig. 2e). In contrast, DUB3 deficiency shortened the half-life of NRF2 (Fig. 2e). Taken together, these data suggest that DUB3 stabilizes NRF2 at the posttranslational level.

\section{DUB3 promotes the transcriptional activity of NRF2}

NRF2, acting as a master transcriptional factor, is stabilized by DUB3. Thus, it is critical to investigate the effect of DUB3 on NRF2 transcriptional activity. To this end, we conducted an ARE-dependent firefly luciferase reporter gene assay. Wild-type DUB3 but not DUB3 ${ }^{\mathrm{C} 89 \mathrm{~S}}$ significantly promoted the transcriptional activity of NRF2 in a dose-dependent manner (Fig. 3a). Under basal conditions, the NRF2 protein level remained relatively low due to proteasomal degradation, so we further confirmed the result with DUB3-shRNAs under tert-butylhydroquinone (tBHQ)induced condition. As expected, DUB3-shRNAs dramatically inhibited the NRF2 transcriptional activity compared with that in the control (Fig. 3b). Moreover, when HEK293T cells were cotransfected with DUB3 and NRF2, the expression of DUB3 and NRF2 synergistically stimulated the ARE reporter (Fig. 3c), indicating that overexpressed NRF2 was continually degraded so that NRF2 expression could not reach saturating level. However, the ability of DUB3 to activate the ARE reporter was almost abolished under NRF2-deficient condition (Fig. 3d). In addition, the mRNA levels of NRF2 downstream genes $H O-1$ and $M R P 2$ changed along with the amount of DUB3 expression (Fig. 3e, f). Our results demonstrate that DUB3 promotes NRF2 transcriptional activity.

\section{DUB3 is in a complex with NRF2 and KEAP1}

To better understand how DUB3 regulates NRF2 and its transcriptional activity, we examined whether DUB3 interacted with NRF2. First, by immunofluorescence staining, 


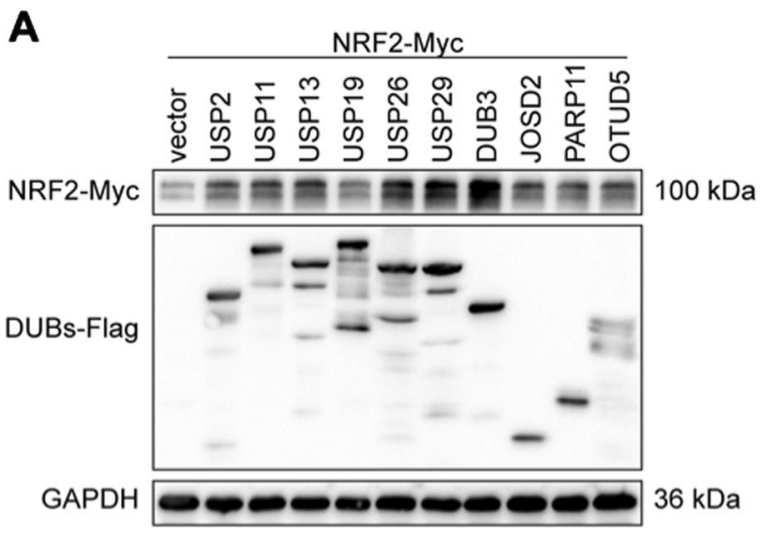

B

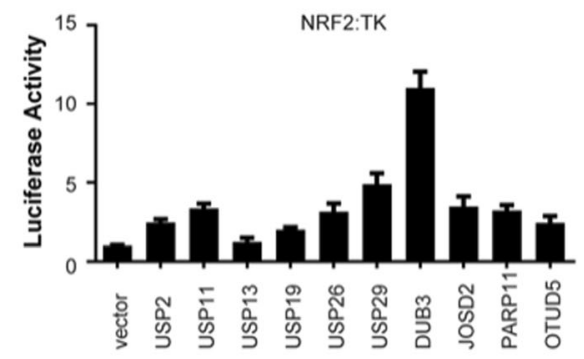

C

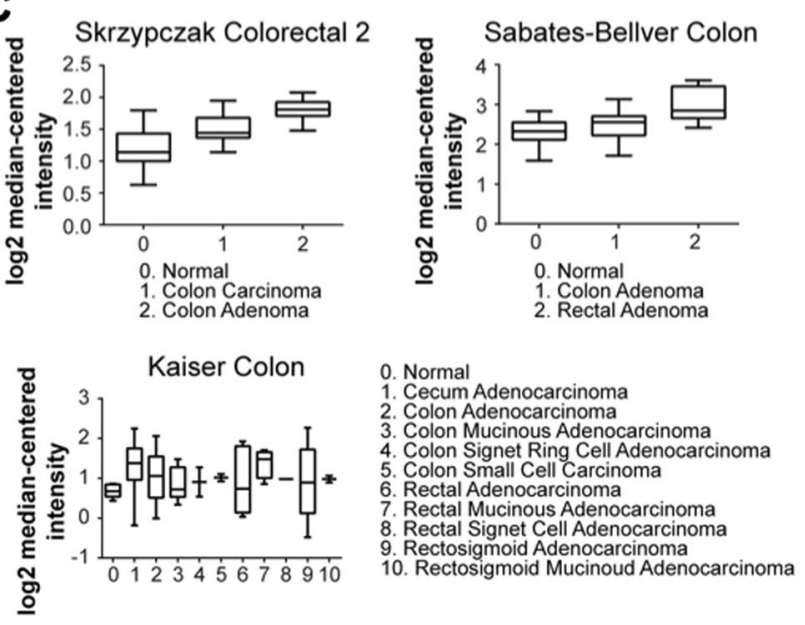

Fig. 1 DUB3 is a potential DUB of NRF2 and correlated expressed with NRF2 in colon cancer. a DUB3 has the strongest effect on NRF2 protein level among the top 10 DUBs. HEK293T cells were cotransfected with NRF2-Myc and individual top 10 DUBs-Flag overexpresiong vectors. Forty-eight hours after transfection, the cell lysates were analyzed by western blot with the indicated antibodies. NRF2Myc protein expression was normalized to GAPDH. b DUB3 has the strongest effect on NRF2 transcriptional activity among the top 10 DUBs. HEK293T cells were cotransfected with ARE reporter firefly luciferase $(100 \mathrm{ng})$, pRL-TK $(10 \mathrm{ng})$ and the indicated amounts of

we found that DUB3-Flag partially colocalized with NRF2HA in U2OS cells (Fig. 4a). Next, we transfected HEK293T cells with DUB3-Flag and NRF2-HA and performed coimmunoprecipitation analysis. Immunoblotting of the HA immunoprecipitate from the cotransfected cells using an anti-Flag antibody showed that DUB3-Flag
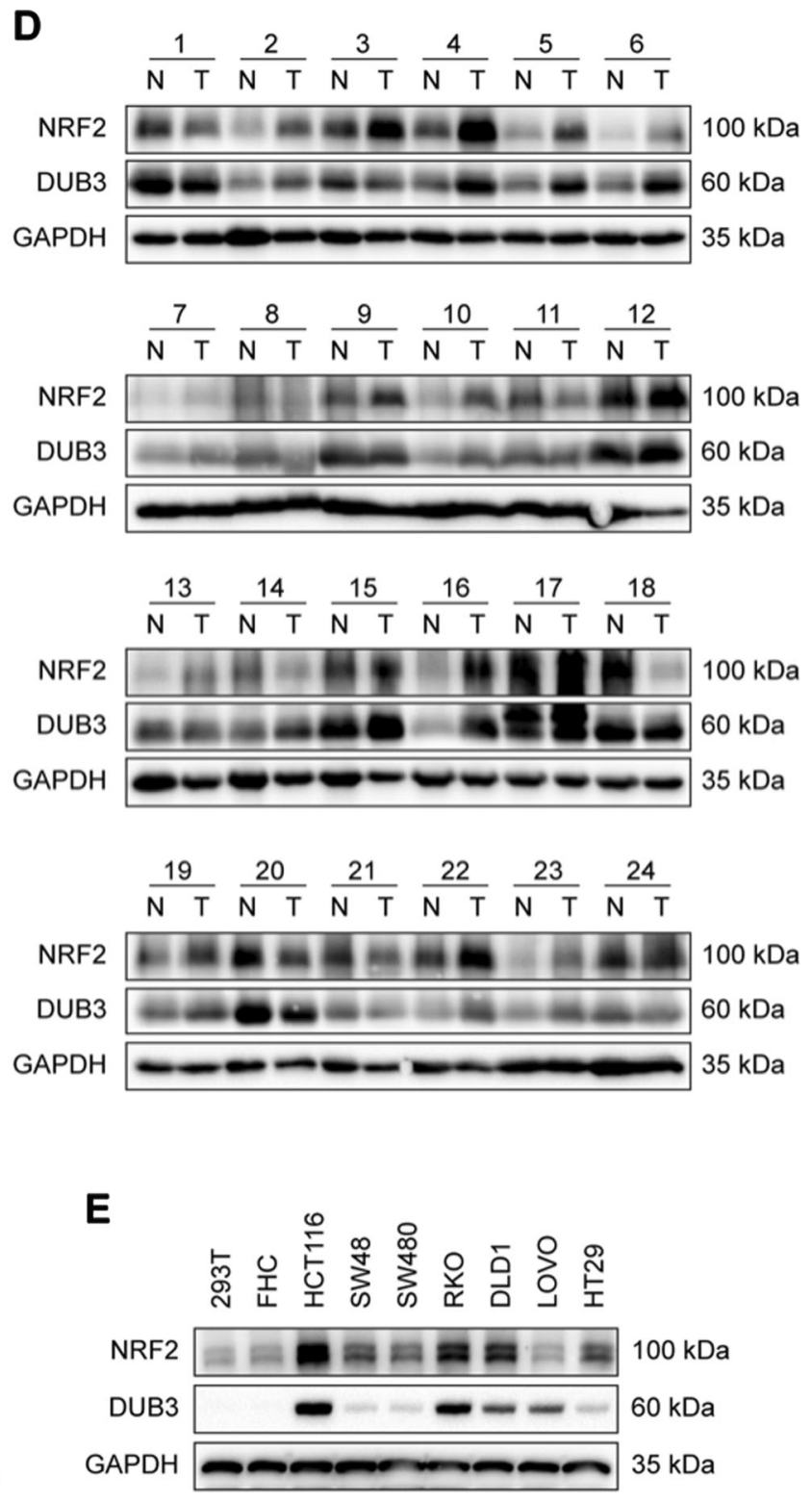

DUBs. Reporter assays were performed $24 \mathrm{~h}$ after transfection, and the results are presented as the NRF2/TK luciferase activity. c Oncomine data mining analysis of $N R F 2$ mRNA levels in three different datasets comparing normal tissues vs. CRC tissues. $\mathbf{d}$ The protein expression of NRF2 and DUB3 in 24 representative pairs of primary CRC (T) and adjacent non-tumor tissues $(\mathrm{N})$. e The protein expression of NRF2 and DUB3 in the human embryonic kidney cell line HEK293T, the normal human colon cell line FHC and seven colorectal cell lines (HCT116, SW48, SW480, RKO, DLD1, LOVO, and HT29)

associated with NRF2-HA (Fig. 4b upper). A similar result was obtained using the Flag immunoprecipitate and antiHA probing (Fig. 4b lower). As KEAP1 is known to interact with NRF2 and to mediate NRF2 ubiquitination by CUL3, we tried to investigate the relationship between DUB3 and KEAP1. In the coimmunoprecipitation 
A

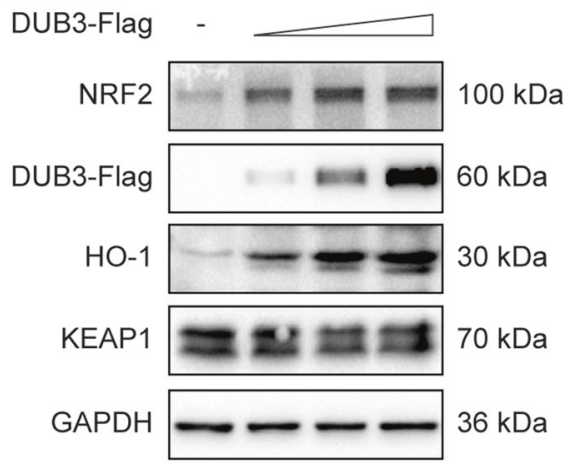

B

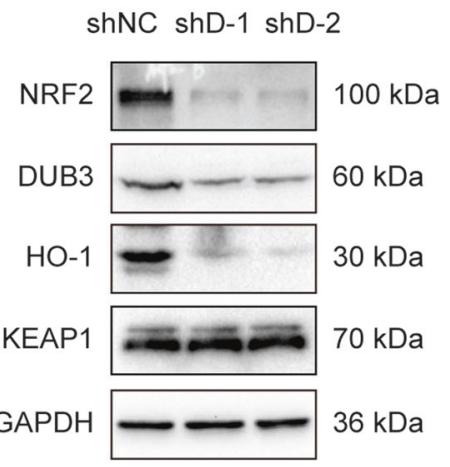

C

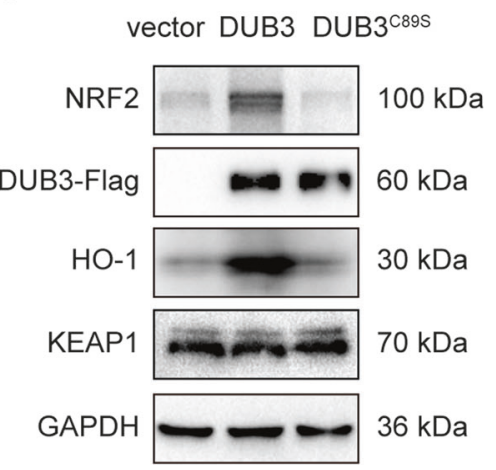

D
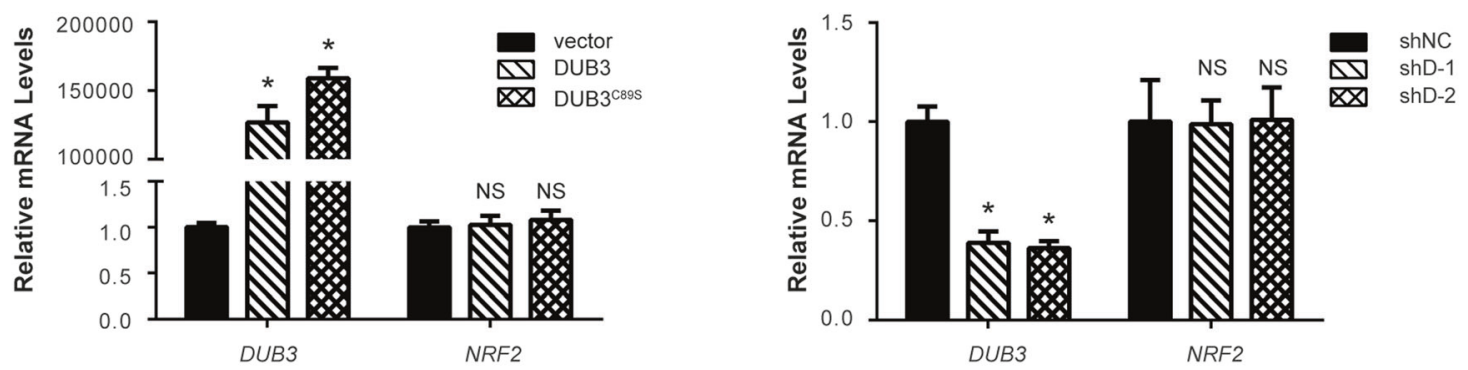

E

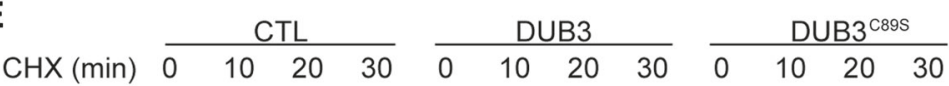

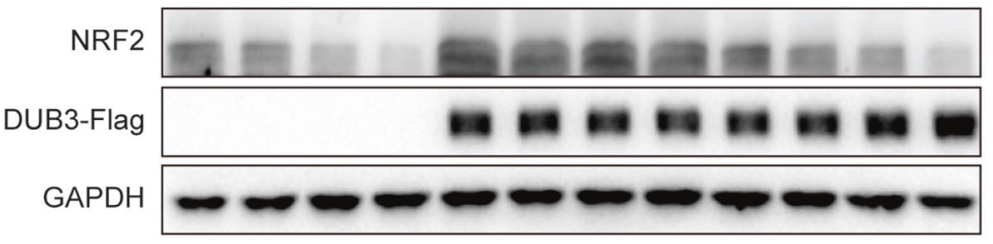

$100 \mathrm{kDa}$

$60 \mathrm{kDa}$

$36 \mathrm{kDa}$

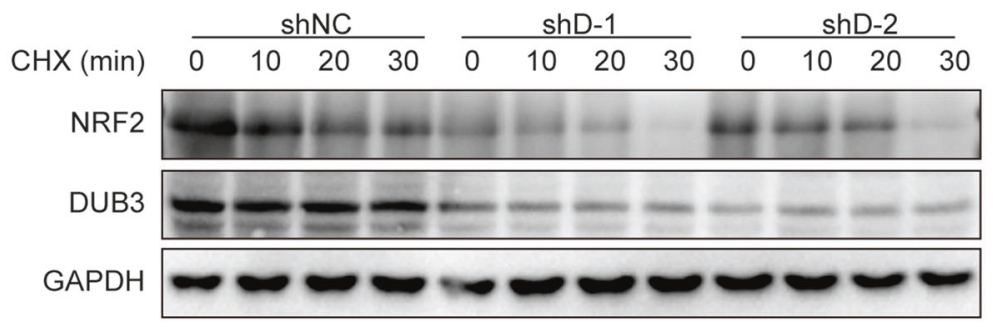

$100 \mathrm{kDa}$
$60 \mathrm{kDa}$
$36 \mathrm{kDa}$

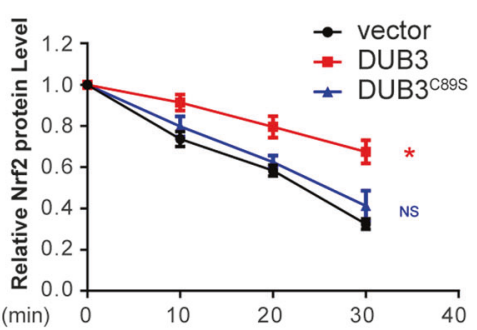

Fig. 2 DUB3 stabilizes NRF2 protein. a Overexpression of DUB3 increases the endogenous NRF2 protein level in a dose-dependent manner. HCT116 cells were transfected with the indicated amounts of DUB3-Flag plasmids. Forty-eight hours after transfection, the cell lysates were analyzed by western blot with the indicated antibodies. The endogenous NRF2 protein expression was normalized to GAPDH. b DUB3 deficiency decreases the endogenous expression of NRF2. HCT116 cells were transfected with the indicated shRNAs. Forty-eight hours after transfection, the cell lysates were analyzed by western blot with the indicated antibodies. c The DUB3 catalytic mutant (DUB3 ${ }^{\mathrm{C} 89 \mathrm{~S}}$ ) lacks the ability to increase NRF2 protein levels. HCT116 cells were transfected with the indicated plasmids. Forty-eight hours after transfection, the cell lysates were analyzed by western blot with

experiment, DUB3-Flag also interacted with KEAP1-HA (Fig. 4c). To further confirm the physiological interaction, we performed endogenous coimmunoprecipitation anti- the indicated antibodies. d DUB3 has no effect on the NRF2 mRNA level. HCT116 cells were transfected with DUB3 expression or shRNA vectors. mRNA was isolated, and qPCR was performed $48 \mathrm{~h}$ after transfection. The NRF2 mRNA level was normalized to GAPDH, and the controls were set equal to 1 . Data were analyzed employing one-way ANOVA and presented as the mean $\pm \mathrm{SD}(N=3$ /group). e The NRF2 half-life is affected by DUB3. Left: HCT116 cells were transfected with the indicated plasmids and treated with $50 \mu \mathrm{g} / \mathrm{mL}$ CHX for the indicated times at $12 \mathrm{~h}$ posttransfection. Cells were harvested for immunoblotting with the indicated antibodies. Right: quantification of the NRF2 protein level normalized to GAPDH. Data were analyzed employing one-way ANOVA and presented as the mean $\pm \mathrm{SD}(N=3$ /group $)$

NRF2 and anti-DUB3, and found that endogenous DUB3 interacted with NRF2 (Fig. 4d). Furthermore, endogenous KEAP1 was also detected in the endogenous NRF2 and 

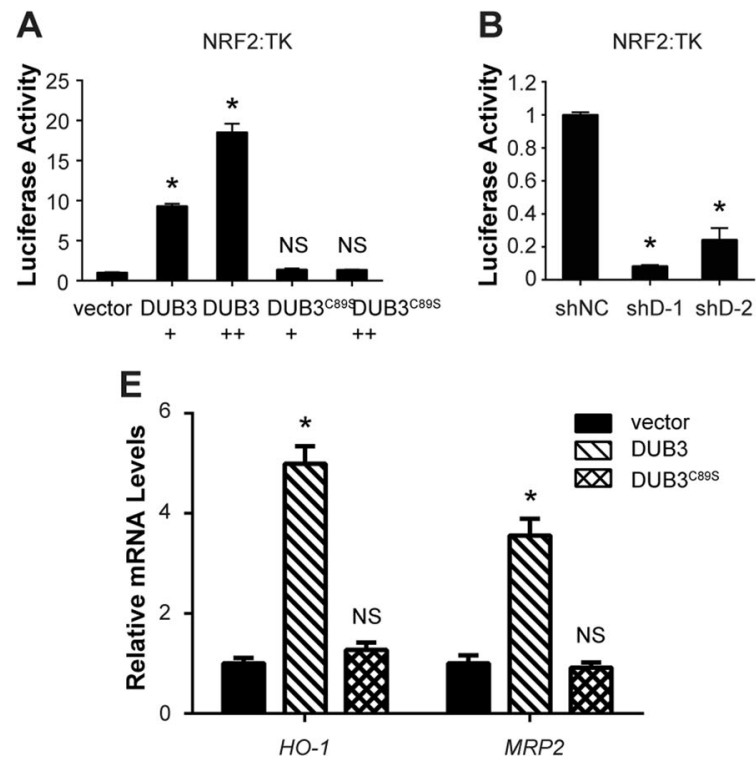

Fig. 3 DUB3 promotes the transcriptional activity of NRF2. a DUB3 but not DUB3 ${ }^{\mathrm{C} 89 \mathrm{~S}}$ activates the transcriptional activity of NRF2. HEK293T cells were cotransfected with ARE reporter firefly luciferase $(100 \mathrm{ng})$, pRL-TK (10 ng) and the indicated amounts of DUB3 or DUB3 ${ }^{\mathrm{C} 9 \mathrm{~S}}$ plasmid. Reporter assays were performed $24 \mathrm{~h}$ after transfection, and the results are presented as the NRF2/TK luciferase activity. Data were analyzed employing one-way ANOVA and presented as the mean \pm SD $(N=3$ /group $)$. b Reduced DUB3 inhibits NRF2 transcriptional activity. The experiments and data analyses were performed as for panel (a). c Synergistic effects of DUB3 and NRF2 on NRF2 transcriptional activation. The experiments were performed as for panel (a). Data were analyzed employing Student's $t$ test and presented as the mean $\pm \mathrm{SD}$ ( $N=3$ /group). d Effects of NRF2

DUB3 IPs (Fig. 4d). These results suggest that DUB3 is in a complex with NRF2 and KEAP1 (Fig. 4e).

\section{DUB3 stabilizes NRF2 via decreasing K48-linked polyubiquitination of NRF2}

To explore the molecular mechanism of NRF2 stabilization by DUB3, pc5-KEAP1-HA was cotransfected with pc5DUB3-Flag into HCT116 cells. The results showed that the DUB3 drastically reduced KEAP1-mediated NRF2 degradation (Fig. 5a). Given that DUB3 interacts with and stabilizes NRF2, we speculated that DUB3 regulated NRF2 through K48-linked deubiquitination. To further investigate the deubiquitinating property of DUB3, a ubiquitination assay was conducted in HCT116 cells. We observed that ectopic expression of DUB3 but not DUB3 ${ }^{\mathrm{C} 89 \mathrm{~S}}$ remarkably reduced KEAP1-mediated NRF2 polyubiquitination (Fig. 5b). In contrast, DUB3 deficiency increased NRF2 polyubiquitination (Fig. 5c), suggesting that the deubiquitinase enzyme activity of DUB3 is indispensable for the DUB3-dependent deubiquitination of NRF2. As NRF2 is known to be conjugated with a K48-linked poly-Ub chain, the K48-only ubiquitin vector was used to examined the
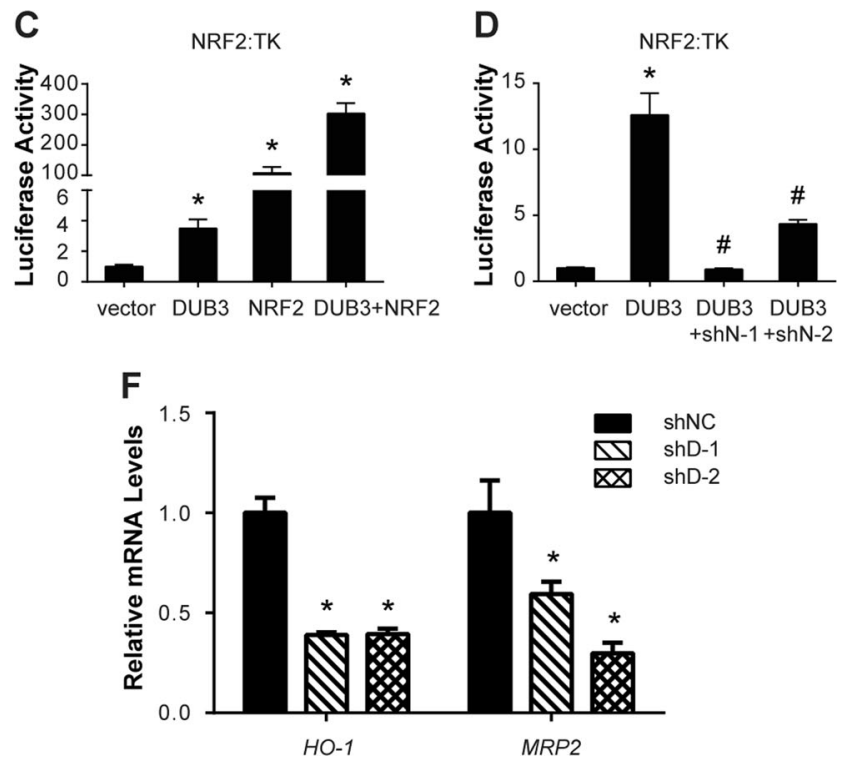

deficiency on the DUB3 induction of NRF2. The experiments were performed as for panel (a). Data were analyzed employing Student's $t$ test and presented as the mean $\pm \mathrm{SD}(N=3$ /group). $* P<0.05$ vs. the empty vector group; ${ }^{\#} P<0.05$ vs. the DUB3 group. e DUB3 overexpression upregulates NRF2 downstream genes. HEK293T cells were transfected with empty vector, DUB3-Flag or DUB3 ${ }^{\text {C } 89}$-Flag. mRNA was isolated, and qPCR was performed $48 \mathrm{~h}$ after transfection. The mRNA levels of the target genes were normalized to $G A P D H$, and controls were set equal to 1 . Data were analyzed employing one-way ANOVA and presented as the mean $\pm \mathrm{SD}(N=3$ /group). f DUB3 deficiency downregulates NRF2 downstream genes. The experiments were performed as for panel (e). Data were analyzed employing oneway ANOVA and presented as the mean $\pm \mathrm{SD}(N=3$ /group)

K48-linked polyubiquitination of NRF2. The results suggested that overexpression of wild-type DUB3 but not ${ }_{\text {DUB }}{ }^{\mathrm{C} 89 \mathrm{~S}}$ reduced NRF2 K48-linked polyubiquitination (Fig. 5d), whereas DUB3 deficiency increased the NRF2 K48-linked polyubiquitination (Fig. 5e). Taken together, these data demonstrate that DUB3 is a bona fide stabilizer of NRF2 through regulation of the K48-linked polyubiquitination of NRF2.

\section{DUB3 causes chemotherapy resistance by upregulating NRF2}

NRF2 overexpression has been found in various types of tumors such as lung [56], breast [57], head and neck [58], ovarian [59], and endometrial cancer [60]. A multitude of studies have shown that high protein expression of NRF2 in cancer cells confers resistance to chemotherapeutic drugs $[61,62]$. Non-small cell lung carcinoma with high level of NRF2 has shown stronger resistance to cisplatin [63]. Doxorubicin-resistant human cancer cells, such as ovarian carcinoma [64] and mammary cancer [65], have shown high levels of NRF2 activity. The above data have clearly demonstrated that DUB3 is a novel DUB that 


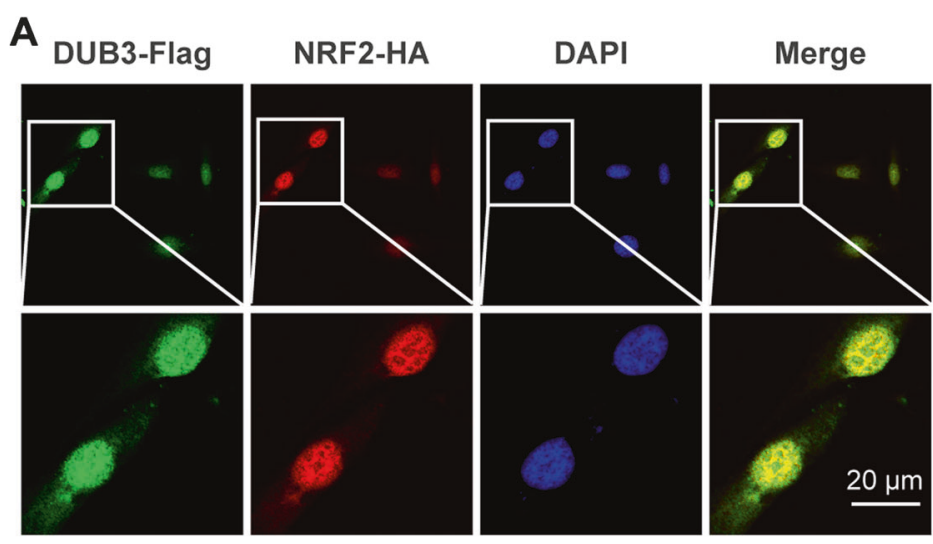

E

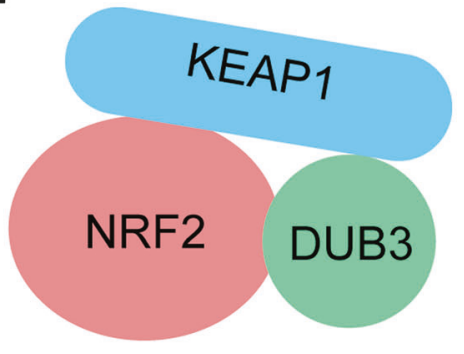

D
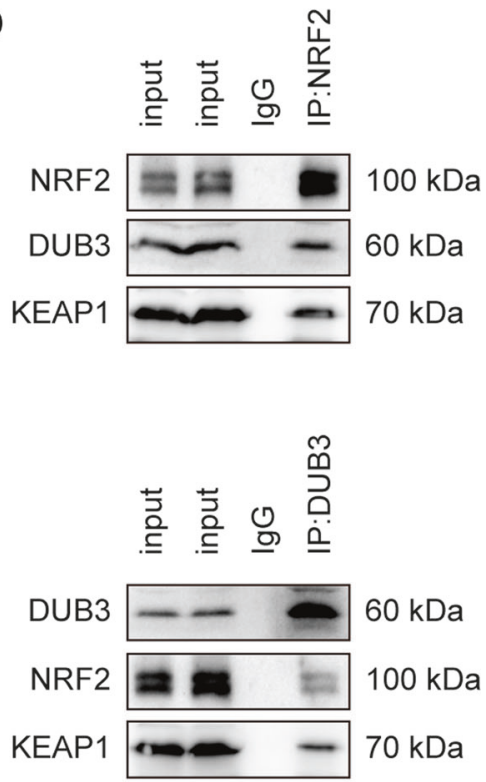

B

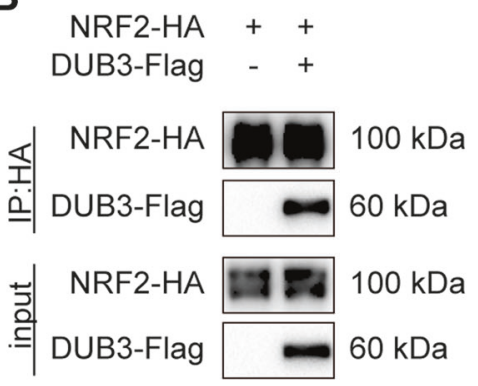

$$
\begin{aligned}
& \text { NRF2-HA - + } \\
& \text { DUB3-Flag }++
\end{aligned}
$$

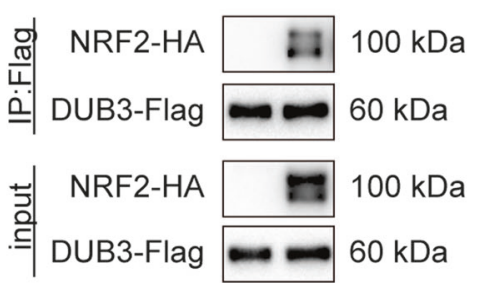

Fig. 4 DUB3 is in a complex with NRF2 and KEAP1. a NRF2 is colocalized with DUB3. U2OS cells were cotransfected with NRF2Myc and DUB3-Flag plasmids. Fluorescence confocal microscopy analysis was performed $36 \mathrm{~h}$ after transfection. b NRF2 interacts with DUB3. HEK293T cells were transfected with the indicated plasmids. Anti-HA or anti-Flag immunoprecipitates were immunoblotted with anti-HA (NRF2) or anti-Flag (DUB3) antibodies, respectively. c

deubiquitinates NRF2 and protects NRF2 from KEAP1dependent degradation. Interestingly, DUB3 is upregulated in many cancers, indicating the potential connection between DUB3 and NRF2 in chemotherapeutic drug resistance in cancer. To prove this notion, three stable HCT116 cell lines, expressing empty vector, NRF2 and DUB3, respectively, were used. We examined the impact of NRF2 and DUB3 on the responses of colon cancer cells to a chemotherapy drug, paclitaxel, by CCK- 8 assay. The paclitaxel concentration required to inhibit cell growth by $50 \%\left(\mathrm{IC}_{50}\right)$ was calculated from viability curves in CCK-8 assay. The $\mathrm{IC}_{50}$ represents cell sensitivity to drugs. Change of $\mathrm{IC}_{50}$ values showed that the HCT116 stable cell lines expressing NRF2 and DUB3 conferred similar paclitaxel resistances (Fig. 6a). More physiologically, NRF2 knockout and DUB3 knockdown HCT116 cell lines were significantly sensitized to paclitaxel treatment (Fig. 6b, c). In addition, the sensitizing effects caused by NRF2 knockout were rescued by re-expression of NRF2 but not DUB3 (Fig. 6d), indicating that DUB3 conferred paclitaxel resistance via NRF2. Furthermore, the paclitaxel resistance effects of NRF2 and DUB3 overexpression and the paclitaxel sensitizing effects of NRF2 and DUB3 deficiency were confirmed by colony formation assay (Fig. 6e-h). To confirm the resistance to chemotherapeutic drugs caused by NRF2, camptothecin was used to examined the impact of 
A

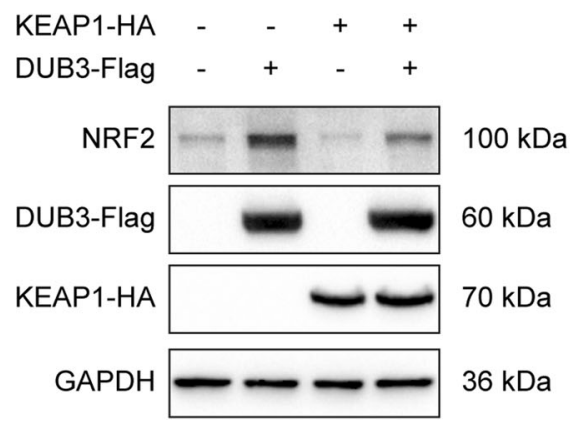

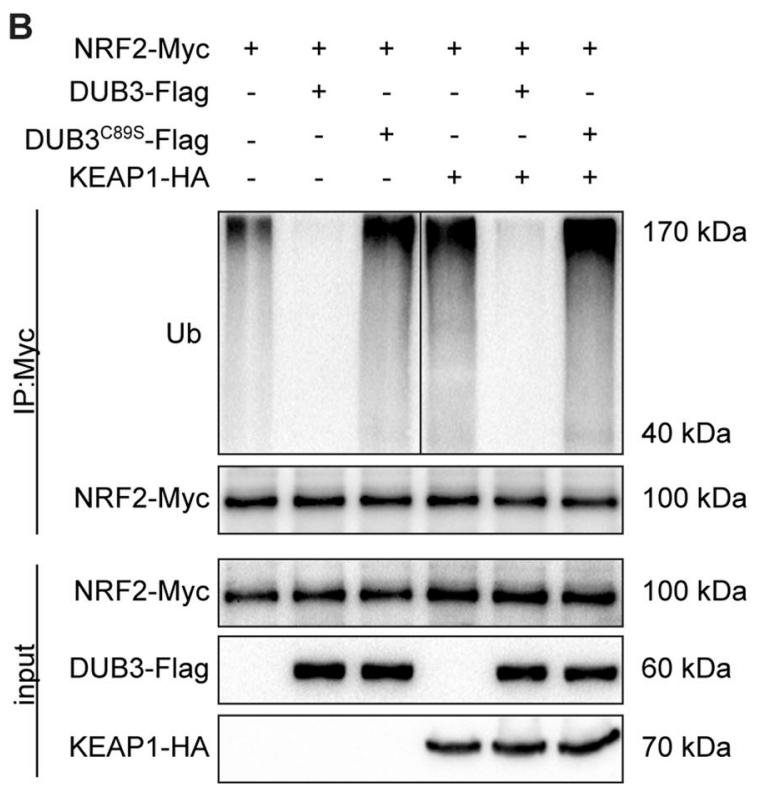

C

NRF2-Myc +++

$\operatorname{shNC}$ shD-1 shD-2

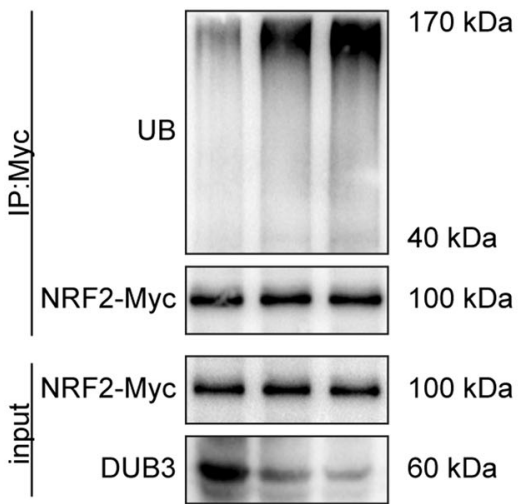

D

$\begin{array}{rrrr}\text { Ub48-HA } & + & + & + \\ \text { NRF2-Myc } & + & + & + \\ \text { DUB3-Flag } & - & + & - \\ \text { B33 }^{\text {C89S-Flag }}- & - & +\end{array}$

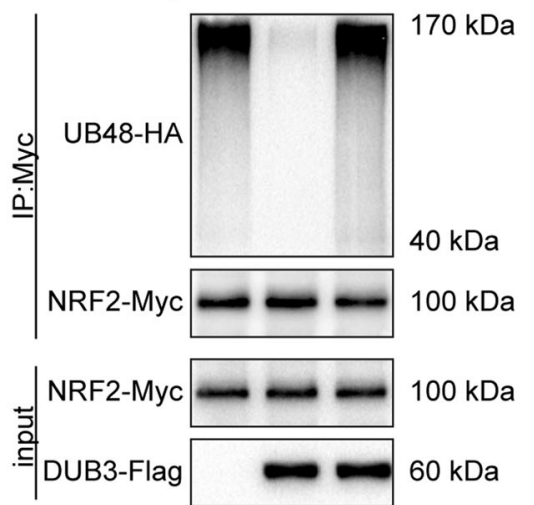

$\mathbf{E}$

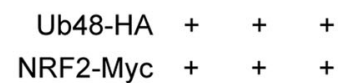

shNC shD-1 shD-2

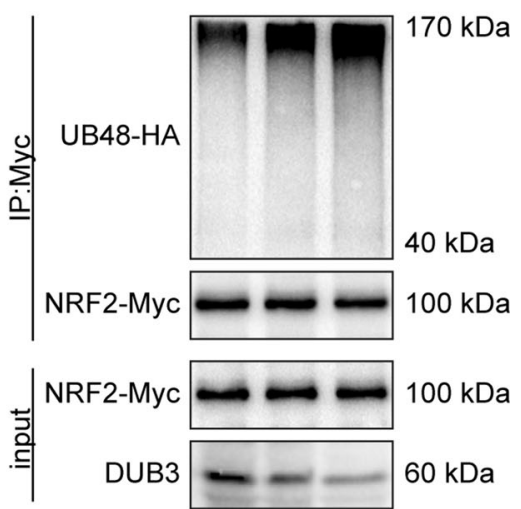

Fig. 5 DUB3 stabilizes NRF2 by decreasing the K48-linked polyubiquitination of NRF2. a DUB3 decreases KEAP1-mediated NRF2 degradation. HCT116 cells were transfected with the indicated plasmids. Forty-eight hours after transfection, the cell lysates were analyzed by western blot with the indicated antibodies. The endogenous NRF2 protein expression was normalized to GAPDH. b DUB3 reduces KEAP1-mediated NRF2 polyubiquitination. HEK293T cells were transfected with the indicated plasmids, and NRF2-Myc was then immunoprecipitated from denatured cell lysates. Cells were treated with $10 \mu \mathrm{M}$ MG132 for $12 \mathrm{~h}$ before collection. c DUB3 depletion increases NRF2 polyubiquitylation. The experiments were performed as for panel (b). d DUB3 decreases the K48-linked polyubiquitylation of NRF2. The K48-only ubiquitin vector was used to examined the K48-linked polyubiquitination of NRF2. The experiments were performed as for panel (b). e DUB3 knockdown increases the K48-linked polyubiquitylation of NRF2. The experiments were performed as for panel (d)

HCT116-NRF2 ${ }^{-/-}$cell line (Fig. 6i). We came to a similar conclusion in RKO experiments (Fig. 6j). The expression of NRF2 and DUB3 in different cell lines was determined by western blot (Fig. S2). Hence, we linked chemotherapy resistance in colon cancer cells to DUB3, the first DUB of NRF2 identified in this study. Taken together, these results demonstrate that DUB3 causes chemotherapy resistance by upregulating NRF2 in colon cancer cells.
DUB3 on HCT116 and RKO cell lines. As expected, the HCT116-NRF2 ${ }^{-1-}$ cell line was more sensitive to camptothecin treatment than HCT116 wild-type cell line, due to the lack of NRF2 (Fig. 6i). Next, we compared the effects of re-expressing NRF2 and DUB3 on camptothecin treatment in the HCT116-NRF2 ${ }^{-l-}$ cell line. Our results demonstrated that DUB3 overexpression had no effect on camptothecin toxicity when compared with NRF2 overexpression in 

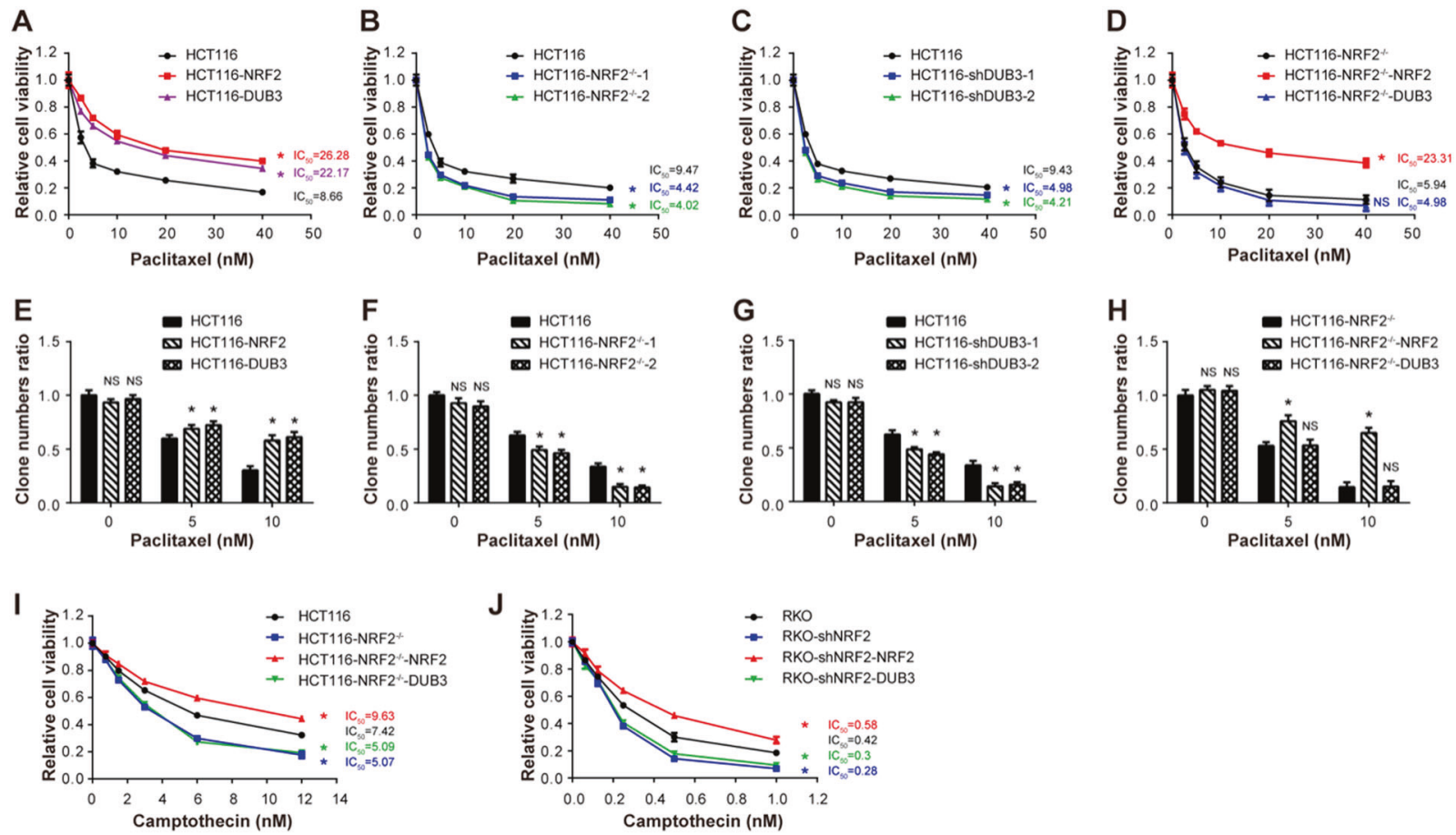

Fig. 6 DUB3 causes chemotherapy resistance by upregulating NRF2 in HCT116 cells. a-d DUB3 causes paclitaxel resistance by upregulating NRF2. We established stable cell lines HCT116-vector, HCT116-NRF2 and HCT116-DUB3, knockout cell lines HCT116$\mathrm{NRF}^{-l-}-1$ and HCT116-NRF2 ${ }^{-1-}-2$, and knockdown cell lines HCT116-shNC, HCT116-shDUB3-1, and HCT116-shDUB3-2. HCT116-NRF2 ${ }^{-I-}$ was infected with lentivirus which contained NRF2 or DUB3 to get the stably rescue cell lines named as HCT116-NRF2 ${ }^{-1-}$-NRF2 and HCT116-NRF2 ${ }^{-I-}$-DUB3. These cell lines were seeded in a 96-well plate and treated with paclitaxel for $48 \mathrm{~h}$ at the indicated

\section{Discussion}

The transduction of the ARE signaling pathway mainly depends on NRF2 and its downstream effector genes, which activate many transcription factors. The NRF2-ARE signaling pathway is closely related to many pathophysiological processes, such as oxidative stress, metabolism, and multidrug resistance. In our study, we chose DUB3 as the most potent candidate from our screening to explore the molecular mechanism by which it regulates the NRF2-ARE signaling pathway in colon cancer cells. We speculated that the redundancy of DUBs might be attributable to the need to match different NRF2 ubiquitination mechanisms mediated by KEAP1, $\beta-\operatorname{TrCP}$, or HRD1. We found that DUB3 interacted with NRF2 and deubiquitinated the Lys-48linked polyubiquitination of NRF2, which ultimately inhibited the proteasomal degradation of NRF2. In addition, we demonstrated the importance of NRF2-ARE signaling in the DUB3-dependent chemotherapy resistance in colon cancer cells.

In recent years, studies by many researchers have clearly shown that NRF2 is polyubiquitinated in Lys-48 mode by doses. Cell viability was measured by CCK-8 assays. e-h DUB3 promotes cell survival after paclitaxel treatment in HCT116 cells. Cells were seeded in a 6-well plate and treated with $5 \mathrm{nM}$ and $10 \mathrm{nM}$ paclitaxel for $48 \mathrm{~h}$ on the following day, after which the medium was replaced with fresh medium. Clones were stained with crystal violet and photographed 10 days later. $\mathbf{i}-\mathbf{j}$ Rescue of DUB3 in NRF2deficient cells cannot recover the resistance to camptothecin in HCT116 and RKO cells. The experiments were performed as for panel (a). Data were analyzed employing one-way ANOVA and presented as the mean $\pm \mathrm{SD}(N=3$ /group $)$

the KEAP1-CUL3 E3 ligase complex and degraded by the $26 \mathrm{~S}$ proteasome. It has also been reported that USP15 negatively regulates NRF2 through the deubiquitination of KEAP1 [14]. Therefore, it seems to be more important to discover the unknown DUBs that directly regulate the K48 ubiquitination and proteasomal degradation of NRF2. In our study, DUB3 interacted with KEAP1 and drastically reduced KEAP1-mediated NRF2 ubiquitination and degradation.

The ubiquitination-proteasome degradation system and the DUB system are of high importance in most cellular processes, including signal transduction, protein degradation, transcriptional regulation, cell cycle regulation, DNA repair, and human diseases and cancer. However, there is no any association between the deubiquitinating enzyme DUB3 and cancer chemotherapy. Based on the link between NRF2 and chemoresistance, we wanted to demonstrate that DUB3 regulates chemoresistance via its ability to deubiquitinate and stabilize NRF2. Our results showed that DUB3 or NRF2 deficiency rendered the colon cancer cell lines HCT116 and RKO more sensitive to paclitaxel and camptothecin. However, rescue by NRF2 but not DUB3 in 
the NRF2-deficient cell lines restored paclitaxel and camptothecin resistance. Taken together, these results suggested that activation of NRF2-ARE signaling might be responsible for the chemotherapy resistance observed in colon cancer cells with overexpressing of DUB3.

The NRF2-ARE antioxidant response pathway plays an important role in chemoprevention and cancer therapy. Consequently, tight regulation is imperative to prevent the onset and progression of cancer. Several studies have shown that cancer cells with high levels of NRF2 are less sensitive to chemotherapy drugs [63-65]. In this report, we identified a novel NRF2 deubiquitinating enzyme, DUB3, and the molecular mechanism of NRF2 regulation by DUB3. This report demonstrates the role of deubiquitination in regulating NRF2-ARE signaling and chemoresistance. Many other candidates were found in our screening test for NRF2 deubiquitinating enzymes. However, the functions, targets, and molecular mechanisms still remain unknown. Thus, our work will provide a good stimulus for further studies on the deubiquitination and regulation mechanisms of NRF2.

Taken together, our results demonstrated that DUB3 caused chemotherapy resistance through NRF2 deubiquitination and stabilization. Our results represent a potentially significant discovery because our data suggest that reagents that antagonize DUB3 could be promising candidates for preventing the colon cancer chemoresistance caused by constitutive activation of NRF2.

Acknowledgements We would like to thank Professor Zhihua Wang for critical reading of the manuscript and Professor Bo Zhong for the Ubiquitin48-HA plasmid. This work was supported by the National Natural Science Foundation of China [31501148, 81470375].

\section{Compliance with ethical standards}

Conflict of interest The authors declare that they have no conflict of interest.

Publisher's note: Springer Nature remains neutral with regard to jurisdictional claims in published maps and institutional affiliations.

\section{References}

1. Siegel RL, Miller KD, Fedewa SA, Ahnen DJ, Meester RGS, Barzi A, et al. Colorectal cancer statistics, 2017. CA Cancer J Clin. 2017;67:177-93.

2. Siegel RL, Miller KD, Jemal A. Cancer statistics, 2018. CA Cancer J Clin. 2018;68:7-30.

3. Trivedi PP, Jena GB, Tikoo KB, Kumar V. Melatonin modulated autophagy and Nrf2 signaling pathways in mice with colitisassociated colon carcinogenesis. Mol Carcinog. 2016;55:255-67.

4. Xu DG, Lv W, Dai CY, Zhu FF, Xu GH, Ma ZJ, et al. 2-(Pro-1ynyl)-5-(5,6-dihydroxypenta-1,3-diynyl) thiophene induces apoptosis through reactive oxygen species-mediated JNK activation in human colon cancer SW620 cells. Anat Rec (Hoboken). 2015;298:376-85.
5. Kruse ML, Friedrich M, Arlt A, Rocken C, Egberts JH, Sebens S, et al. Colonic lamina propria inflammatory cells from patients with IBD induce the nuclear factor-E2 related factor-2 thereby leading to greater proteasome activity and apoptosis protection in human colonocytes. Inflamm Bowel Dis. 2016;22:2593-606.

6. Stachel I, Geismann C, Aden K, Deisinger F, Rosenstiel P, Schreiber S, et al. Modulation of nuclear factor E2-related factor-2 (Nrf2) activation by the stress response gene immediate early response-3 (IER3) in colonic epithelial cells: a novel mechanism of cellular adaption to inflammatory stress. J Biol Chem. 2014;289:1917-29.

7. Sebens S, Bauer I, Geismann C, Grage-Griebenow E, Ehlers S, Kruse ML, et al. Inflammatory macrophages induce Nrf2 transcription factor-dependent proteasome activity in colonic NCM460 cells and thereby confer anti-apoptotic protection. J Biol Chem. 2011;286:40911-21.

8. Kim TH, Hur EG, Kang SJ, Kim JA, Thapa D, Lee YM, et al. NRF2 blockade suppresses colon tumor angiogenesis by inhibiting hypoxia-induced activation of HIF- $1 \alpha$. Cancer Res. 2011;71:2260-75.

9. Longley DB, Harkin DP, Johnston PG. 5-fluorouracil: mechanisms of action and clinical strategies. Nat Rev Cancer. 2003;3:330-8.

10. Verma RP, Hansch C. Camptothecins: a SAR/QSAR study. Chem Rev. 2009;109:213-35.

11. Chen M, Liang X, Gao C, Zhao R, Zhang N, Wang S, et al. Ultrasound triggered conversion of porphyrin/camptothecinfluoroxyuridine triad microbubbles into nanoparticles overcomes multidrug resistance in colorectal cancer. ACS Nano. 2018;12:7312-26.

12. Kang KA, Piao MJ, Kim KC, Kang HK, Chang WY, Park IC, et al. Epigenetic modification of Nrf2 in 5-fluorouracil-resistant colon cancer cells: involvement of TET-dependent DNA demethylation. Cell death \& Dis. 2014;5:e1183.

13. Zhao XQ, Zhang YF, Xia YF, Zhou ZM, Cao YQ. Promoter demethylation of nuclear factor-erythroid 2-related factor 2 gene in drug-resistant colon cancer cells. Oncol Lett. 2015;10:1287-92.

14. Villeneuve NF, Tian W, Wu T, Sun Z, Lau A, Chapman E, et al. USP15 negatively regulates Nrf2 through deubiquitination of Keap1. Mol Cell. 2013;51:68-79.

15. Hayes JD, Dinkova-Kostova AT. The Nrf2 regulatory network provides an interface between redox and intermediary metabolism. Trends Biochem Sci. 2014;39:199-218.

16. Ma Q. Role of nrf2 in oxidative stress and toxicity. Annu Rev Pharmacol Toxicol. 2013;53:401-26.

17. Harvey CJ, Thimmulappa RK, Sethi S, Kong X, Yarmus L, Brown $\mathrm{RH}$, et al. Targeting Nrf2 signaling improves bacterial clearance by alveolar macrophages in patients with COPD and in a mouse model. Sci Transl Med. 2011;3:78ra32.

18. Paek J, Lo JY, Narasimhan SD, Nguyen TN, Glover-Cutter K, Robida-Stubbs S, et al. Mitochondrial SKN-1/Nrf mediates a conserved starvation response. Cell Metab. 2012;16:526-37.

19. Mahaffey CM, Zhang H, Rinna A, Holland W, Mack PC, Forman HJ. Multidrug-resistant protein-3 gene regulation by the transcription factor Nrf2 in human bronchial epithelial and non-smallcell lung carcinoma. Free Radic Biol \& Med. 2009;46:1650-7.

20. Cuadrado A, Manda G, Hassan A, Alcaraz MJ, Barbas C, Daiber A, et al. Transcription factor NRF2 as a therapeutic target for chronic diseases: a systems medicine approach. Pharmacol Rev. 2018;70:348-83.

21. Sporn MB, Liby KT. NRF2 and cancer: the good, the bad and the importance of context. Nat Rev Cancer. 2012;12:564-71.

22. Rojo de la Vega M, Chapman E, Zhang DD. NRF2 and the hallmarks of cancer. Cancer Cell 2018;21-43. 
23. Furukawa M, Xiong Y. BTB protein Keap1 targets antioxidant transcription factor Nrf2 for ubiquitination by the Cullin 3-Roc1 ligase. Mol Cell Biol. 2005;25:162-71.

24. Lo SC, Li X, Henzl MT, Beamer LJ, Hannink M. Structure of the Keap1:Nrf2 interface provides mechanistic insight into Nrf2 signaling. EMBO J. 2006;25:3605-17.

25. Dinkova-Kostova AT, Kostov RV, Canning P. Keap1, the cysteine-based mammalian intracellular sensor for electrophiles and oxidants. Arch Biochem Biophys. 2017;617:84-93.

26. Hur W, Gray NS. Small molecule modulators of antioxidant response pathway. Curr Opin Chem Biol. 2011;15:162-73.

27. Rachakonda G, Xiong Y, Sekhar KR, Stamer SL, Liebler DC, Freeman ML. Covalent modification at Cys151 dissociates the electrophile sensor Keap1 from the ubiquitin ligase CUL3. Chem Res Toxicol. 2008;21:705-10.

28. Baird L, Dinkova-Kostova AT. Diffusion dynamics of the Keap1Cullin3 interaction in single live cells. Biochem Biophys Res Commun. 2013;433:58-65.

29. Zhang DD, Lo SC, Sun Z, Habib GM, Lieberman MW, Hannink M. Ubiquitination of Keap1, a BTB-Kelch substrate adaptor protein for $\mathrm{Cul3}$, targets Keap1 for degradation by a proteasomeindependent pathway. J Biol Chem. 2005;280:30091-9.

30. Kobayashi A, Kang MI, Okawa H, Ohtsuji M, Zenke Y, Chiba T, et al. Oxidative stress sensor Keap1 functions as an adaptor for Cul3-based E3 ligase to regulate proteasomal degradation of Nrf2. Mol Cell Biol. 2004;24:7130-9.

31. Chowdhry S, Zhang Y, McMahon M, Sutherland C, Cuadrado A, Hayes JD. Nrf2 is controlled by two distinct beta-TrCP recognition motifs in its Neh6 domain, one of which can be modulated by GSK-3 activity. Oncogene. 2013;32:3765-81.

32. Wu T, Zhao F, Gao B, Tan C, Yagishita N, Nakajima T, et al. Hrd1 suppresses Nrf2-mediated cellular protection during liver cirrhosis. Genes Dev. 2014;28:708-22.

33. Burrows JF, McGrattan MJ, Rascle A, Humbert M, Baek KH, Johnston JA. DUB-3, a cytokine-inducible deubiquitinating enzyme that blocks proliferation. J Biol Chem. 2004;279:139934000 .

34. Burrows JF, McGrattan MJ, Johnston JA. The DUB/USP17 deubiquitinating enzymes, a multigene family within a tandemly repeated sequence. Genomics. 2005;85:524-9.

35. Zhu Y, Pless M, Inhorn R, Mathey-Prevot B, D'Andrea AD. The murine DUB-1 gene is specifically induced by the betac subunit of interleukin-3 receptor. Mol Cell Biol. 1996;16:4808-17.

36. Baek KH, Kim MS, Kim YS, Shin JM, Choi HK. DUB-1A, a novel deubiquitinating enzyme subfamily member, is polyubiquitinated and cytokine-inducible in B-lymphocytes. J Biol Chem. 2004;279:2368-76.

37. Zhu Y, Lambert K, Corless C, Copeland NG, Gilbert DJ, Jenkins NA, et al. DUB-2 is a member of a novel family of cytokineinducible deubiquitinating enzymes. J Biol Chem. 1997;272:51-7.

38. Baek KH, Mondoux MA, Jaster R, Fire-Levin E, D'Andrea AD. DUB-2A, a new member of the DUB subfamily of hematopoietic deubiquitinating enzymes. Blood. 2001;98:636-42.

39. Saitoh Y, Miyamoto N, Okada T, Gondo Y, Showguchi-Miyata J, Hadano S, et al. The RS447 human megasatellite tandem repetitive sequence encodes a novel deubiquitinating enzyme with a functional promoter. Genomics. 2000;67:291-300.

40. Shin JM, Yoo KJ, Kim MS, Kim D, Baek KH. Hyaluronan- and RNA-binding deubiquitinating enzymes of USP17 family members associated with cell viability. BMC Genom. 2006;7:292.

41. Pereg Y, Liu BY, O'Rourke KM, Sagolla M, Dey A, Komuves L, et al. Ubiquitin hydrolase Dub3 promotes oncogenic transformation by stabilizing Cdc25A. Nat Cell Biol. 2010;12:400-6.

42. Delgado-Diaz MR, Martin Y, Berg A, Freire R, Smits VA. Dub3 controls DNA damage signalling by direct deubiquitination of H2AX. Mol Oncol. 2014;8:884-93.
43. Kim J, D’Annibale S, Magliozzi R, Low TY, Jansen P, Shaltiel IA, et al. USP17- and SCF $\beta$ TrCP-regulated degradation of DEC1 controls the DNA damage response. Mol Cell Biol. 2014;34:4177-85.

44. Chen R, Zhang L, Zhong B, Tan B, Liu Y, Shu HB. The ubiquitin-specific protease 17 is involved in virus-triggered type I IFN signaling. Cell Res. 2010;20:802-11.

45. Nguyen HT, Kugler JM, Cohen SM. DUB3 deubiquitylating enzymes regulate hippo pathway activity by regulating the stability of ITCH, LATS and AMOT proteins. PLOS ONE. 2017;12: e0169587.

46. Shaw JA, Page K, Blighe K, Hava N, Guttery D, Ward B, et al. Genomic analysis of circulating cell-free DNA infers breast cancer dormancy. Genome Res. 2012;22:220-31.

47. McFarlane C, Kelvin AA, de la Vega M, Govender U, Scott CJ, Burrows JF, et al. The deubiquitinating enzyme USP17 is highly expressed in tumor biopsies, is cell cycle regulated, and is required for G1-S progression. Cancer Res. 2010;70:3329-39.

48. McFarlane C, McFarlane S, Paul I, Arthur K, Scheaff M, Kerr K, et al. The deubiquitinating enzyme USP17 is associated with nonsmall cell lung cancer (NSCLC) recurrence and metastasis. Oncotarget. 2013;4:1836-43.

49. Zhou B, Shu B, Xi T, Su N, Liu J. Dub3 expression correlates with tumor progression and poor prognosis in human epithelial ovarian cancer. Biomed Pharmacother. 2015;70:84-9.

50. Song C, Liu W, Li J. USP17 is upregulated in osteosarcoma and promotes cell proliferation, metastasis, and epithelialmesenchymal transition through stabilizing SMAD4. Tumour Biol: J Int Soc Oncodev Biol Med. 2017;39:1010428317717138.

51. Cong L, Zhang F. Genome engineering using CRISPR-Cas9 system. Methods Mol Biol. 2015;1239:197-217.

52. Zhang ZY, Li SZ, Zhang HH, Wu QR, Gong J, Liang T, et al. Stabilization of ATF5 by TAK1-Nemo-like kinase critically regulates the interleukin-1 $\beta$-stimulated $\mathrm{C} / \mathrm{EBP}$ signaling pathway. Mol Cell Biol. 2015;35:778-88.

53. Zhang HH, Li SZ, Zhang ZY, Hu XM, Hou PN, Gao L, et al. Nemo-like kinase is critical for p53 stabilization and function in response to DNA damage. Cell Death Differ. 2014;21:1656-63.

54. Yamamoto M, Kensler TW, Motohashi H. The KEAP1-NRF2 system: a thiol-based sensor-effector apparatus for maintaining redox homeostasis. Physiol Rev. 2018;98:1169-203.

55. Rhodes DR, Yu J, Shanker K, Deshpande N, Varambally R, Ghosh D, et al. ONCOMINE: a cancer microarray database and integrated data-mining platform. Neoplasia. 2004;6:1-6.

56. Shibata T, Ohta T, Tong KI, Kokubu A, Odogawa R, Tsuta K, et al. Cancer related mutations in NRF2 impair its recognition by Keap1-Cul3 E3 ligase and promote malignancy. Proc Natl Acad Sci USA. 2008;105:13568-73.

57. Nioi P, Nguyen T. A mutation of Keap1 found in breast cancer impairs its ability to repress Nrf2 activity. Biochem Biophys Res Commun. 2007;362:816-21.

58. Stacy DR, Ely K, Massion PP, Yarbrough WG, Hallahan DE, Sekhar KR, et al. Increased expression of nuclear factor E2 p45related factor 2 (NRF2) in head and neck squamous cell carcinomas. Head Neck. 2006;28:813-8.

59. van der Wijst MG, Brown R, Rots MG. Nrf2, the master redox switch: the Achilles' heel of ovarian cancer? Biochim Biophys Acta. 2014;1846:494-509.

60. Jiang T, Chen N, Zhao F, Wang XJ, Kong B, Zheng W, et al. High levels of Nrf2 determine chemoresistance in type II endometrial cancer. Cancer Res. 2010;70:5486-96.

61. Singh A, Misra V, Thimmulappa RK, Lee H, Ames S, Hoque $\mathrm{MO}$, et al. Dysfunctional KEAP1-NRF2 interaction in non-smallcell lung cancer. PLoS Med. 2006;3:e420.

62. Saito T, Ichimura Y, Taguchi K, Suzuki T, Mizushima T, Takagi $\mathrm{K}$, et al. p62/Sqstm1 promotes malignancy of HCV-positive 
hepatocellular carcinoma through Nrf2-dependent metabolic reprogramming. Nat Commun. 2016;7:12030.

63. Homma S, Ishii Y, Morishima Y, Yamadori T, Matsuno Y, Haraguchi N, et al. Nrf2 enhances cell proliferation and resistance to anticancer drugs in human lung cancer. Clin Cancer Res. 2009; 15:3423-32.

64. Shim GS, Manandhar S, Shin DH, Kim TH, Kwak MK. Acquisition of doxorubicin resistance in ovarian carcinoma cells accompanies activation of the NRF2 pathway. Free Radic Biol \& Med. 2009;47:1619-31.

65. Zhong Y, Zhang F, Sun Z, Zhou W, Li ZY, You QD, et al. Drug resistance associates with activation of Nrf2 in MCF-7/ DOX cells, and wogonin reverses it by down-regulating Nrf2-mediated cellular defense response. Mol Carcinog. 2013;52:824-34. 